\title{
The dilemma: How to assure quality in surgical clinics
}

$\mathrm{C}$ linic surgery is increasing. The ease of booking surgery, the ability to choose talented staff and the excellence of modern anesthesia make clinic surgery an exciting new trend for the 21 st century. The quality of surgery performed in surgical clinics is excellent and easily equals the quality of surgery performed in general hospitals. To assure that quality remains high, clinic operators should seek membership in a surgical clinic association as a means of continuing education and quality assurance. Clinic inspections held on a regular basis provide objective reviews by peer surgeons, and offer suggestions and problem solving advice. Membership involves examining a number of charts on a routine basis for completeness, keeping an untoward occurrence book, and complying with safety standards and fire codes, as well providing emergency devices and drugs for resuscitation. It is the clinic operator's responsibility to aspire to the highest standard of care. Voluntary membership stresses the motivational aspect of safety.

A decade ago, these associations adopted a bylaw stating that surgeons performing surgery in clinics should have privileges to perform the same procedures in general hospitals. There were several reasons for this. It provided a way to ascertain that the hospital department of surgery had reviewed the surgeon's training and recent experience in specific operations, and provided a seamless method of ensuring that the patient would be likely to receive postsurgical care from the operating surgeon in the event of an urgent complication or by another surgeon taking call for him or her should he or she be away or off call. In actuality, most clinic-based surgeons plan surgery so as to be easily available for a number of days after surgery because it is far easier for the patient to call the operating surgeon than to call someone else.

Although this bylaw makes sense, in practice, there are a number of problems, including the following:

- Hospitals are no more competent in determining surgical competency than are individuals. There is, however, an advantage of having a bigger organizational structure to observe new operations and assess training before performing the operation. In 1984 when I saw liposuction presented at a national meeting, I returned and immediately began doing the operation. A cannula was just another instrument, and the technique was simple. What made the procedure safe was that I was a fully trained, practising plastic surgeon who understood wound care, surgical trauma and resuscitation. Without full training, as signified by my FRCSC after obtaining an MD, I would not have been able to use a cannula safely. This is true of any fully trained plastic surgeon doing a new operation in his or her specialty.

Some procedures are outside the hospital realm to consider doing and, therefore, are outside the competence of hospitals to have a valid opinion regarding surgical competency, eg, hair transplantation, laser skin resurfacing and chemical peels.

- The issue is important because some clinic surgeons do not have an active hospital appointment; only those with active appointments are allowed by the hospital to do elective (nonemergency) surgery. So only those who have active appointments are assessed for the ability to perform the same surgery that they perform in clinics. Those with courtesy appointments are not allowed to do elective surgery, so a courtesy appointment is of no use in quality assurance for clinic surgeons.

- There are numerous reasons why some plastic surgeons do not have active staff appointments. An active appointment involves a considerable commitment, and sometimes the commitment is too onerous. The main commitment is emergency call, and although it is important for the patients, our specialty's reputation and our relationships with our colleagues in other specialties, sometimes the trade is just not worth it. By the trade I mean the exchange of emergency call (onerous) for operating time (desirable). Or the emergency call (too many days) is not balanced by operating time (too few hours). Emergency call needs to be better recompensed, but none of us think that will happen, so the burden causes some clinic surgeons to decline active hospital appointments.

Some clinic surgeons think that they do not need to be on hospital staffs because patients have a right to be treated at any hospital due to medicare and can, therefore, easily get treated for complications. Some surgeons think that they do not need a hospital appointment because the patient will need a specialist in another field such as internal medicine, but 
they may have forgotten that some patients may have a surgical complication such as a large hematoma, may require the services of a general hospital and may need to be re-operated upon to drain the hematoma and find and control the bleeding vessel. Ideally, the operating surgeon should be the one to do this. If the operating surgeon is not available, another surgeon in the same specialty on call will assist the patient. However, that surgeon has no chance of having his or her favour to the operating surgeon and patient repaid if the operating surgeon does not have an active appointment somewhere.

- Some operations are best done in hospitals because they are extensive or prolonged. These patients are more likely to need nursing care and have postoperative bleeding. We surgeons need to ask ourselves "what is the safest thing to do"? It follows that there are some patients who should not be operated on in clinics and discharged the same day because it is not the safest thing to do. Patients having large reduction mammaplasties, large abdomenoplasties and liposuction aspirations over $5000 \mathrm{~cm}^{3}$ are better admitted to hospitals for these reasons. California and Florida have adopted a rule that liposuction patients (if done in a clinic) should have no more than $5000 \mathrm{~cm}^{3}$ aspirated and that those who have more than $5000 \mathrm{~cm}^{3}$ aspirated should be operated on in a hospital. Why? Is a hospital safer? Does it make it easier to admit the patient after surgery because the patient is already there? Why not say that all patients in whom more than $5000 \mathrm{~cm}^{3}$ is going to be aspirated should be admitted to hospital before surgery?
As well, performing multiple procedures at the same operation should be rethought if a case can be made for increased safety by staging or performing those procedures with lighter anesthetic techniques.

- Patients come long distances for surgery. If they are operated on in clinics, surgeons should consider whether the patient should return home after surgery or stay somewhere near the clinic overnight. Travel the day of surgery can be detrimental and increases the chance that the patient will be seen late with a postoperative emergency. These patients can be considered to be itinerant. We should think the same way about them as we do about itinerant surgeons - that it has its own set of problems and requires careful management.

Therefore, there is a dilemma as to how to assure quality in surgical clinics that are outside the purview of hospital medical advisory and quality assurance committees. This is not a critical issue because FRCSC surgeons are fully trained with such a wide surgical view that seemingly new procedures performed are not really new but rather the natural evolution of operations that the surgeon is currently performing. FRCSC surgeons have an excellent grasp of the ethical practice of medicine, which put simply is, "what is the safest thing to do"? and "am I the best person to do it?"

John R Taylor jrtaylor@inforamp.net 